\title{
Species richness in restinga vegetation on the eastern Maranhão State, Northeastern Brazil
}

\author{
Flávia Cristina Vieira SERRA ${ }^{1}$, Patrícia Barbosa LIMA², Eduardo Bezerra de ALMEIDA Jr. ${ }^{3^{*}}$ \\ 1 Universidade Federal do Maranhão. Programa de Pós-graduação em Biodiversidade e Conservação, Departamento de Biologia, Av. dos Portugueses, 1966, Bacanga, 65085- \\ 580, São Luís, MA, Brasil. \\ 2 Universidade Federal Rural de Pernambuco. Programa de Pós-Graduação em Botânica. Av. Dom Manoel de Medeiros s/n, Dois Irmãos, 52171-900, Recife, PE, Brasil. \\ ${ }^{3}$ Universidade Federal do Maranhão. Laboratório de Estudos Botânicos, Departamento de Biologia, Av. dos Portugueses, 1966, Bacanga, 65085-580, São Luís, MA, Brasil. \\ * Corresponding author: ebaj25@yahoo.com.br
}

\begin{abstract}
The State of Maranhão comprises the second largest coastline in Brazil. Nonetheless, few floristic surveys on restinga vegetation have been performed in Northeastern Brazil. This study aimed to survey floristic in a restinga of Maranhão, and to assess its similarity to other restingas in the states of Pará and Piauí. Botanical specimens were sampled at Sítio Aguahy, in the municipality of São José de Ribamar, along the eastern coast of Maranhão between June/2012 and October/2013. Species identification and life forms classification followed the usual methods employed in floristic studies. Similarities in plant composition of six sites were estimated based on cluster analysis through Bray-Curtis distance. In the studied area, we found 116 phanerogamic species, 100 genera and 52 families; 19 species were new occurrences for the restinga of Maranhão. Families with largest number of species were Fabaceae, Cyperaceae, Rubiaceae, Poaceae, Myrtaceae, Asteraceae, Malvaceae, Combretaceae, and Eriocaulaceae, which comprised $49.2 \%$ of all sampled species. We identified 41 nanophanerophytes, 34 terophytes, 15 camephytes, ten microphanerophytes, six hemicryptophytes, and five lianas. Similarity analyses indicated greater affinity between the restinga flora from the states of Maranhão and Pará than that observed for the states of Maranhão and Piauí, suggesting that the process of species colonization in the former is more likely derived from the Amazon Forest. It is expected that these results may encourage future researchers to conduct further surveys in restinga areas of the Maranhão aiming to better understand the influence of neighboring vegetation on the colonization of the coastal areas of Maranháo.
\end{abstract}

KEYWORDS: Coastal sand plains, ecotone, floristic similarity, Brazilian northeast.

\section{Riqueza de espécies em uma restinga no leste do Maranhão, Nordeste do Brasil}

\section{RESUMO}

O Estado do Maranhão possui o segundo maior litoral da costa brasileira. No entanto, poucos levantamentos florísticos foram realizados nas áreas de restinga no Nordeste do Brasil. O presente estudo teve como objetivo realizar um levantamento florístico em uma área de restinga no Maranháo e analisar a similaridade com a flora das restingas dos Estados do Pará e do Piauí. As coletas botânicas foram realizadas na restinga do Sítio Aguahy, município de São José de Ribamar, na porção oriental da Ilha do Maranhão, no período de junho de 2012 a outubro de 2013. A identificação das espécies e a classificação das formas de vida seguiu a metodologia usual em estudos florísticos. A similaridade foi realizada através de análise de cluster a partir da distância de Bray-Curtis, onde foram considerados seis estudos. Foram listadas 116 espécies fanerogâmicas, 100 gêneros e 52 famílias. Deste total, 19 espécies são apresentadas como novas ocorrências para o litoral do Maranhão. As famílias com maior número de espécies foram Fabaceae, Cyperaceae, Rubiaceae, Poaceae, Myrtaceae, Asteraceae, Malvaceae, Combretaceae e Eriocaulaceae, correspondendo a 49.2\% das espécies encontradas. Quanto às formas de vida, foram identificados 41 nanofanerófitos, 34 terófitos, 15 caméfitos, 10 microfanerófitos, seis hemicriptófitos e cinco lianas. A análise de similaridade mostrou maior afinidade entre a flora da restinga do Maranhão e do Pará, do que com a flora do Piauí, sugerindo uma possível colonização da restinga estudada por espécies provenientes da floresta Amazônica. Espera-se que esses dados sejam vistos como um passo inicial para a realização de mais pesquisas nas demais restingas maranhenses para que, futuramente, proporcione a compreensão da influência da vegetação circunvizinha na colonização das áreas litorâneas do Maranhão.

PALAVRAS-CHAVE: Planície arenosa costeira, ecótono, similaridade florística, Nordeste do Brasil. 


\section{INTRODUCTION}

Restinga ecosystems (coastal vegetation) are considered by some researchers (Araujo and Henriques 1984; Souza et al. 2008) as a type of vegetation that from coastal tropical and subtropical coastal areas, with individuals present in herbaceous, shrubby, and arboreous layers, growing on sandy plains dating from Quaternary period. These coastal ecosystems are currently threatened due to a diverse range of anthropogenic interference, such as deforestation, real estate speculation, and land occupation (Santos-Filho et al. 2013), thus demanding urgent conservation actions (Dias and Soares 2008). Thus, conservation efforts on this type of vegetation are needed. The efforts should be associated with initiatives that try to understand its biodiversity and the ecological interactions among species on the restinga, particularly considering the high plasticity of plant species in these areas (Scarano 2002).

Northeastern Brazil restinga floristic composition was explored in several studies (Sacramento et al. 2007; Cantarelli et al. 2012; Santos-Filho et al. 2013). Although Maranhão has the second largest coastline in the country $(640 \mathrm{~km})$ (El-Robrini et al. 2006), up to date, only Cabral-Freire and Monteiro (1993) examined this vegetation closely.

Previous studies suggest that southeastern and northeastern Brazil restinga vegetation could be an extension of the Atlantic Forest (Scarano 2002; Santos-Filho et al. 2013). However, surveys carried out on the Amazonian coast (Amaral et al. 2008) observed that species distribution in these coastal restinga sites are dissimilar to those of southeastern Brazil, thus highlighting the necessity for additional studies in the Amazon coast and more comparisons amongst floras of neighboring areas in eastern northeast Brazil.

Floristic assessment are fundamental to determine richness of a given area, thenceforth providing essential information to ecological studies related to composition and structure vegetation (Chaves et al. 2013). The aims of this study were to perform a floristic survey of an area of restinga vegetation on Maranhão Island and to evaluate its floristic similarity to other restinga sites of the neighboring States.

\section{MATERIALS AND METHODS}

Plants were sampled in the restinga of Sítio Aguahy (02038'47”S, 4409'05”W), an area owned by Companhia Farmacêutica Quercegen Agronegócios I Ltda., in the municipality of São José de Ribamar, of the Maranhão State, Brazil; a map of the study site is available elsewhere (Araujo et al. 2016). The Sítio Aguahy comprises an area of 400 ha with transitional vegetation coverage, combining fragments of regenerating Amazon Forest, mangrove swamps, and restinga (vegetation with marine influence, according to the IBGE 2012). The examined restinga site covers approximately 50 ha, with herbaceous and shrub-arboreal vegetation.
Regional climate has two distinct seasons: a rainy season from January to June, and a dry season from July to December. The local climate is classified as Aw type (Köppen 1948). Annual mean rainfall of the Maranhão State is about 1,250 $2,000 \mathrm{~mm}$, and mean temperatures throughout the year vary between $25.5^{\circ} \mathrm{C}$ and $28.6^{\circ} \mathrm{C}$ (INMET 2015).

\section{Sampling and identification of botanical material}

Botanical sampling was carried out between June/2012 and October/2013. We selected specimens with viable reproductive structures, during walk through permanent and implanted trails in study area, with the purpose of increased sampling effort.

After collection, the plant material was dried and then analyzed based on methods suggested by Mori et al. (1989). Plants were identified to species level based on specialized literature. Family classification followed the proposed system in APG III (2009). Species and author names were confirmed by consulting List of Species of the Brazilian Flora (2014).

The classification and grouping of life forms followed the criteria of Raunkiaer (1934) adapted by Ellenberg and Mueller-Dombois (1967). After identification, specimens were incorporated into the MAR herbarium at the Department of Biology, Federal University of Maranhão (Universidade Federal do Maranhão - UFMA).

Description of phytophysiognomy followed classification of Silva and Britez (2005), in which type of formation is related to predominant plant life forms, besides differentiating physiognomies based on the flood regime of water table. This method was previously employed to determine restinga main features in northeastern Brazil (Sacramento et al. 2007; Almeida Jr. et al. 2009; Santos-Filho et al. 2010).

\section{Data Analysis}

We used grouping analyses based on the Unweighted Pair Group Method with Arithmetic Mean (UPGMA) from Jaccard distance index (Table 1) to evaluate similarity between different restinga areas. Additionally, a dendrogram was generated based on cophenetic correlation index, using Pearson's correlation. Cut off limit for grouping method was based on Borcard et al. (2011), observing a predetermined number of groups (in this case, $n=3$ ). These analyses were performed using Vegan package (Oksanen et al. 2013) of the $\mathrm{R}$ software version 3.1.1 (R Development Core Team 2013).

\section{RESULTS}

We identified 116 species, 100 genera and 52 families (Table 2). Families represented by largest numbers of species were Fabaceae (with 18 species; $15.3 \%$ of total); Cyperaceae (9; 7.6\%); Rubiaceae (8; 6.8\%); Myrtaceae (5; 4.2\%); Poaceae (4; 3.4\%), Asteraceae (4; 3.4\%), Malvaceae (4; 3.4\%), 
Table 1. Restingas areas of the Brazilian north-northeast used in the Jaccard similarity analysis.

\begin{tabular}{|c|c|c|c|c|c|}
\hline Area & State & Coordinates & $\begin{array}{l}\text { No of } \\
\text { Species }\end{array}$ & Physiognomies & Reference \\
\hline Sítio Aguahy & Maranhão (MA) & $02^{\circ} 38^{\prime} 47^{\prime \prime} \mathrm{S}, 4^{\circ} 09^{\prime} 05^{\prime \prime} \mathrm{W}$ & 116 & fields, shrub and forest & Present study \\
\hline Ilha Grande & Piauí (PI) & $02^{\circ} 50^{\prime} 84^{\prime \prime} \mathrm{S}, 41^{\circ} 47^{\prime} 39^{\prime \prime} \mathrm{W}$ & 67 & fields, shrub and carnaubais & Santos-Filho et al. (2015) \\
\hline Parnaíba & Piauí (PI) & $02^{\circ} 55^{\prime} 48^{\prime \prime} \mathrm{S}, 41^{\circ} 40^{\prime} 67^{\prime \prime} \mathrm{W}$ & 131 & fields, shrub & Santos-Filho et al. (2013) \\
\hline Luiz Correia & Piauí (PI) & $02^{\circ} 55^{\prime} 89^{\prime \prime} \mathrm{S}, 41^{\circ} 30^{\prime} 49^{\prime \prime} \mathrm{W}$ & 116 & restinga forest & Santos-Filho et al. (2013) \\
\hline Algodoal 1 & Pará (PA) & $00^{\circ} 36^{\prime} 57,6^{\prime \prime} \mathrm{S}, 47^{\circ} 32^{\prime} 41,7^{\prime \prime} \mathrm{W}$ & 171 & dunes & Santos and Rosário (1988) \\
\hline Algodoal 2 & Pará (PA) & $00^{\circ} 34^{\prime} 45^{\prime \prime} \mathrm{S}, 47^{\circ} 34^{\prime} 12^{\prime \prime} \mathrm{W}$ & 224 & $\begin{array}{l}\text { "psamófila reptante", "brejo- } \\
\text { herbáceo", field dunes, } \\
\text { shrub fields, and forest }\end{array}$ & Bastos et al. (1995) \\
\hline Maiandeua & Pará (PA) & 00³4'30"S, 47034'12”W & 57 & $\begin{array}{l}\text { shrub restinga, and herbaceous } \\
\text { restinga }\end{array}$ & Bastos (1988) \\
\hline
\end{tabular}

Table 2. List of species recorded in the Sítio Aguahy Restinga, municipality of São José de Ribamar, Maranhão State, Brazil. The species marked with an asterisk ${ }^{*}$ ) represent the first record for the Maranhão Restingas.

\begin{tabular}{|c|c|c|}
\hline Family / Species & Life forms & Collector / number \\
\hline \multicolumn{3}{|l|}{ Aizoaceae } \\
\hline Sesuvium portulacastrum (L.) L. & Terophyte & Serra, F.C.V. 168 \\
\hline \multicolumn{3}{|l|}{ Alstroemeriaceae } \\
\hline Bomarea edulis (Tussac) Herb. & Terophyte & Serra, F.C.V. 184 \\
\hline \multicolumn{3}{|l|}{ Amaranthaceae } \\
\hline Alternanthera brasiliana (L.) Kuntze & Terophyte & Serra, F.C.V. 125 \\
\hline Blutaparon portulacoides (A. St.-Hil.) Mears & Terophyte & Serra, F.C.V. 154 \\
\hline \multicolumn{3}{|l|}{ Anacardiaceae } \\
\hline Anacardium occidentale L. & Nanophanerophyte & Serra, F.C.V. 65 \\
\hline Tapirira obtusa (Benth.) J.D. Mitch. & Microphanerophyte & Serra, F.C.V. 110 \\
\hline \multicolumn{3}{|l|}{ Annonaceae } \\
\hline Annona glabra L. & Nanophanerophyte & Serra, F.C.V. 105 \\
\hline Duguetia surinamensis R.E.Fr. * & Nanophanerophyte & Almeida Jr., E.B. 890 \\
\hline \multicolumn{3}{|l|}{ Apocynaceae } \\
\hline Rhabdadenia biflora (Jacq.) Müll.Arg. & Liana & Serra, F.C.V. 104 \\
\hline \multicolumn{3}{|l|}{ Arecaceae } \\
\hline Astrocaryum vulgare Mart. & Nanophanerophyte & Serra, F.C.V. 128 \\
\hline \multicolumn{3}{|l|}{ Asteraceae } \\
\hline Emilia sonchifolia (L.) DC. & Terophyte & Serra, F.C.V. 192 \\
\hline Rolandra fruticosa (L.) Kuntze & Nanophanerophyte & Serra, F.C.V. 72 \\
\hline Vernonanthura brasiliana (L.) H.Rob. & Nanophanerophyte & Serra, F.C.V. 94 \\
\hline Wedelia villosa Gardner * & Terophyte & Serra, F.C.V. 175 \\
\hline \multicolumn{3}{|l|}{ Bignoniaceae } \\
\hline Bignonia aequinoctialis L. & Liana & Serra, F.C.V. 145 \\
\hline \multicolumn{3}{|l|}{ Bixaceae } \\
\hline Cochlospermum orinocense (Kunth) Steud. & Microphanerophyte & Serra, F.C.V. 83 \\
\hline \multicolumn{3}{|l|}{ Boraginaceae } \\
\hline Euploca polyphylla (Lehm.) J.I.M.Melo \& Semir & Hemicryptophyte & Serra, F.C.V. 170 \\
\hline Varronia globosa Jacq. & Nanophanerophyte & Serra, F.C.V. 20 \\
\hline \multicolumn{3}{|l|}{ Cactaceae } \\
\hline Cereus jamacaru DC. & Nanophanerophyte & Almeida Jr., E.B. 891 \\
\hline \multicolumn{3}{|l|}{ Cannabaceae } \\
\hline Trema micrantha (L.) Blume & Nanophanerophyte & Serra, F.C.V. 140 \\
\hline \multicolumn{3}{|l|}{ Capparaceae } \\
\hline Cynophalla flexuosa (L.) J.PresI * & Nanophanerophyte & Serra, F.C.V. 141 \\
\hline
\end{tabular}


Table 2. Continuation

\begin{tabular}{|c|c|c|}
\hline Family / Species & Life forms & Collector / number \\
\hline \multicolumn{3}{|l|}{ Chrysobalanaceae } \\
\hline Chrysobalanus icaco L. & Nanophanerophyte & Serra, F.C.V. 08 \\
\hline Hirtella racemosa Lam. & Nanophanerophyte & Almeida Jr., E.B. 892 \\
\hline \multicolumn{3}{|l|}{ Combretaceae } \\
\hline Conocarpus erectus $\mathrm{L}$. & Nanophanerophyte & Serra, F.C.V. 163 \\
\hline Terminalia argentea Mart. & Nanophanerophyte & Almeida Jr., E.B. 912 \\
\hline Terminalia glabrescens Mart. & Nanophanerophyte & Serra, F.C.V. 149 \\
\hline \multicolumn{3}{|l|}{ Commelinaceae } \\
\hline Dichorisandra hexandra (Aubl.) C.B.Clarke* & Terophyte & Almeida Jr., E.B. 893 \\
\hline \multicolumn{3}{|l|}{ Convolvulaceae } \\
\hline Merremia tuberosa (L.) Rendle & Terophyte & Almeida Jr., E.B. 906 \\
\hline \multicolumn{3}{|l|}{ Cyperaceae } \\
\hline Cyperus laxus Lam. & Terophyte & Serra, F.C.V. 159 \\
\hline Cyperus sp. & Terophyte & Serra, F.C.V. 172 \\
\hline Eleocharis geniculata (L.) Roem. \& J. Schull. & Terophyte & Almeida Jr., E.B. 914 \\
\hline Eleocharis sp. & Terophyte & Serra, F.C.V. 102 \\
\hline Fiurena umbellata Rottb. & Terophyte & Serra, F.C.V. 21 \\
\hline Kyllinga sp. & Terophyte & Serra, F.C.V. 100 \\
\hline Pycreus polystachyos (Rottb.) P.Beauv. & Terophyte & Almeida Jr., E.B. 894 \\
\hline Rhynchospora sp. & Terophyte & Serra, F.C.V. 87 \\
\hline Scleria bracteata Cav. & Terophyte & Serra, F.C.V. 113 \\
\hline \multicolumn{3}{|l|}{ Eriocaulaceae } \\
\hline Paepalanthus bifidus Scharde * & Hemicryptophyte & Almeida Jr. E.B. 937 \\
\hline Paepalanthus lamarckii Kunth & Hemicryptophyte & Serra, F.C.V. 196 \\
\hline Paepalanthus sublitis Miq. & Hemicryptophyte & Almeida Jr., E. B. 936 \\
\hline \multicolumn{3}{|l|}{ Erythroxylaceae } \\
\hline Erythroxylum passerinum Mart. & Nanophanerophyte & Almeida Jr., E. B. 920 \\
\hline \multicolumn{3}{|l|}{ Fabaceae } \\
\hline Abarema cochleata (Willd.) Barneby \& J.W.Grimes & Microphanerophyte & Serra, F.C.V. 115 \\
\hline Aeschynomene brevipes Benth. & Camephyte & Serra, F.C.V. 171 \\
\hline Aeschynomene fluminensis Vell. & Camephyte & Serra, F.C.V. 42 \\
\hline Centrosema brasilianum (L.) Benth. & Vine & Serra, F.C.V. 12 \\
\hline Centrosema aff. pubescens Benth. & Vine & Serra, F.C.V. 178 \\
\hline Chamaecrista diphylla (L.) Greene & Hemicryptophyte & Serra, F.C.V. 177 \\
\hline Chamaecrista flexuosa (L.) Greene & Hemicryptophyte & Serra, F.C.V. 59 \\
\hline Chloroleucon acacioides (Ducke) Barneby \& J.W.Grimes & Microphanerophyte & Serra, F.C.V. 97 \\
\hline Copaifera langsdorffii Desf. * & Nanophanerophyte & Serra, F.C.V. 54 \\
\hline Crotalaria retusa L. & Terophyte & Serra, F.C.V. 40 \\
\hline Desmodium triflorum (L.) DC. & Camephyte & Serra, F.C.V. 29 \\
\hline Dioclea reflexa Hook. f. * & Liana & Serra, F.C.V. 56 \\
\hline Erythrina amazonica Krukoff & Nanophanerophyte & Serra, F.C.V. 49 \\
\hline Guilandina bonduc L. & Nanophanerophyte & Serra, F.C.V. 151 \\
\hline Hymenaea courbaril L. & Microphanerophyte & Serra, F.C.V. 43 \\
\hline Stylosanthes angustifolia Vogel & Camephyte & Serra, F.C.V. 91 \\
\hline Zornia guanipensis Pittier & Camephyte & Serra, F.C.V. 30 \\
\hline Zornia reticulata Sm. & Camephyte & Almeida Jr., E.B. 943 \\
\hline \multicolumn{3}{|l|}{ Gentianaceae } \\
\hline Schultesia guianensis (Aubl.) Malme & Terophyte & Serra, F.C.V. 77 \\
\hline \multicolumn{3}{|l|}{ Heliconiaceae } \\
\hline Heliconia psittacorum L. f. & Terophyte & Serra, F.C.V. 74 \\
\hline \multicolumn{3}{|l|}{ Hidroleaceae } \\
\hline Hydrolea spinosa L. & Terophyte & Serra, F.C.V. 143 \\
\hline
\end{tabular}


Table 2. Continuation

\begin{tabular}{|c|c|c|}
\hline Family / Species & Life forms & Collector / number \\
\hline \multicolumn{3}{|l|}{ Hypericaceae } \\
\hline Vismia guianensis (Aubl.) Pers & Nanophanerophyte & Serra, F.C.V. 119 \\
\hline \multicolumn{3}{|l|}{ Lamiaceae } \\
\hline Amasonia campestris (Aubl.) Moldenke & Camephyte & Serra, F.C.V. 164 \\
\hline Marsypianthes chamaedrys (Vahl) Kuntze & Terophyte & Serra, F.C.V. 26 \\
\hline \multicolumn{3}{|l|}{ Lauraceae } \\
\hline Cassytha filiformis $\mathrm{L}$. & Parasite & Almeida Jr., E.B. 895 \\
\hline \multicolumn{3}{|l|}{ Lecythidaceae } \\
\hline Gustavia augusta L. & Microphanerophyte & Serra, F.C.V. 144 \\
\hline \multicolumn{3}{|l|}{ Lentibulariaceae } \\
\hline Utricularia fimbriata Kunth * & Terophyte & Almeida Jr., E.B. 1015 \\
\hline Utricularia nigrescens Sylvén * & Terophyte & Almeida Jr., E.B. 1016 \\
\hline \multicolumn{3}{|l|}{ Loganiaceae } \\
\hline Spigelia anthelmia L. & Terophyte & Serra, F.C.V. 64 \\
\hline \multicolumn{3}{|l|}{ Loranthaceae } \\
\hline Psittacanthus sp. & Hemiparasite & Serra, F.C.V. 163 \\
\hline \multicolumn{3}{|l|}{ Malpighiaceae } \\
\hline Banisteriopsis muricata (Cav.) Cuatrec. & Nanophanerophyte & Serra, F.C.V. 45 \\
\hline Byrsonima crassifolia (L.) Kunt & Microphanerophyte & Serra, F.C.V. 76 \\
\hline \multicolumn{3}{|l|}{ Malvaceae } \\
\hline Helicteres heptandra L.B.Sm & Nanophanerophyte & Serra, F.C.V. 160 \\
\hline Pavonia sp. & Nanophanerophyte & Serra, F.C.V. 85 \\
\hline Sida ciliaris L. & Camephyte & Serra, F.C.V. 96 \\
\hline Sida cordifolia L. & Nanophanerophyte & Serra, F.C.V. 37 \\
\hline \multicolumn{3}{|l|}{ Melastomataceae } \\
\hline Mouriri guianensis Aubl. & Nanophanerophyte & Serra, F.C.V. 203 \\
\hline Nepsera aquatica (Aubl.) Naudin & Camephyte & Serra, F.C.V. 69 \\
\hline Tococa guianensis Aubl. & Nanophanerophyte & Serra, F.C.V. 207 \\
\hline \multicolumn{3}{|l|}{ Myrtaceae } \\
\hline Campomanesia sp. & Nanophanerophyte & Serra, F.C.V. 78 \\
\hline Eugenia sp. 1 & Nanophanerophyte & Serra, F.C.V. 50 \\
\hline Eugenia sp. 2 & Nanophanerophyte & Serra, F.C.V. 139 \\
\hline Myrcia sylvatica (G. Mey.) DC. * & Nanophanerophyte & Serra, F.C.V. 118 \\
\hline Myrciaria cf. cuspidata 0. Berg * & Nanophanerophyte & Serra, F.C.V. 103 \\
\hline \multicolumn{3}{|l|}{ Nyctaginaceae } \\
\hline Guapira opposita (Vell.) Reitz & Nanophanerophyte & Serra, F.C.V. 185 \\
\hline \multicolumn{3}{|l|}{ Ochnaceae } \\
\hline Ouratea fieldingiana (Gardner) Engl. * & Microphanerophyte & Serra, F.C.V. 112 \\
\hline Sauvagesia erecta L. & Camephyte & Serra, F.C.V. 80 \\
\hline \multicolumn{3}{|l|}{ Onagraceae } \\
\hline Ludwigia hyssopifolia (G. Don) Exell * & Camephyte & Serra, F.C.V. 38 \\
\hline Ludwigia sp. & Camephyte & Almeida Jr., E.B. 987 \\
\hline \multicolumn{3}{|l|}{ Olacaceae } \\
\hline Heisteria ovata Benth. & Nanophanerophyte & Serra, F.C.V. 82 \\
\hline \multicolumn{3}{|l|}{ Plantaginaceae } \\
\hline Stemodia foliosa Benth. * & Terophyte & Serra, F.C.V. 58 \\
\hline \multicolumn{3}{|l|}{ Poaceae } \\
\hline Brachiaria sp. & Terophyte & Almeida Jr., E.B. 909 \\
\hline Eriochloa sp. & Terophyte & Serra, F.C.V. 15 \\
\hline Hymenachne pernambucensis (Spreng.) Zuloaga & Terophyte & Serra, F.C.V. 102 \\
\hline Penissetum sp. & Terophyte & Serra, F.C.V. 62 \\
\hline \multicolumn{3}{|l|}{ Polygalaceae } \\
\hline Polygala paniculata L. & Terophyte & Serra, F.C.V. 179 \\
\hline Polygala violacea Aubl. & Terophyte & Serra, F.C.V. 181 \\
\hline
\end{tabular}


Table 2. Continuation

\begin{tabular}{|c|c|c|}
\hline Family / Species & Life forms & Collector / number \\
\hline \multicolumn{3}{|l|}{ Polygonaceae } \\
\hline Coccoloba mollis Casar. & Nanophanerophyte & Serra, F.C.V. 133 \\
\hline Coccoloba ramosissima Wedd. & Nanophanerophyte & Serra, F.C.V. 93 \\
\hline \multicolumn{3}{|l|}{ Rubiaceae } \\
\hline Borreria verticillata (L.) G.Mey. & Camephyte & Serra, F.C.V. 95 \\
\hline Chiococca nitida Benth. & Nanophanerophyte & Serra, F.C.V. 57 \\
\hline Duroia macrophylla Huber * & Nanophanerophyte & Serra, F.C.V. 84 \\
\hline Faramea nitida Benth & Nanophanerophyte & Almeida Jr., E.B. 990 \\
\hline Guettarda angelica Mart. ex Müll.Arg. & Nanophanerophyte & Serra, F.C.V. 174 \\
\hline Isertia spiciformis DC. & Nanophanerophyte & Serra, F.C.V. 152 \\
\hline Mitracarpus salzmannianus DC. * & Camephyte & Serra, F.C.V. 47 \\
\hline Psychotria hoffmannseggiana (Willd. ex Schult.) Müll.Arg. & Nanophanerophyte & Serra, F.C.V. 153 \\
\hline \multicolumn{3}{|l|}{ Santalaceae } \\
\hline Phoradendron quadrangulare (Kunth) Griseb. & Hemiparasite & Almeida Jr., E.B. 872 \\
\hline \multicolumn{3}{|l|}{ Sapindaceae } \\
\hline Serjania salzmanniana Schltdl. * & Liana & Serra, F.C.V. 132 \\
\hline \multicolumn{3}{|l|}{ Sapotaceae } \\
\hline Manilkara bidentata (A. DC.) A. Chev. * & Microphanerophyte & Serra, F.C.V. 106 \\
\hline Manilkara triflora (Allemão) Monach. * & Microphanerophyte & Serra, F.C.V. 32 \\
\hline \multicolumn{3}{|l|}{ Smilacaceae } \\
\hline Smilax sp. & Liana & Almeida Jr., E.B. 896 \\
\hline \multicolumn{3}{|l|}{ Violaceae } \\
\hline Pombalia calceolaria (L.) Paula-Souza & Camephyte & Serra, F.C.V. 48 \\
\hline \multicolumn{3}{|l|}{ Vitaceae } \\
\hline Cissus erosa Rich. & Liana & Serra, F.C.V. 156 \\
\hline \multicolumn{3}{|l|}{ Xyridaceae } \\
\hline Xyris jupicai Rich. & Terophyte & Serra, F.C.V. 01 \\
\hline
\end{tabular}

Combretaceae $(3 ; 2.5 \%)$, and Eriocaulaceae $(3 ; 2.5 \%)$. These families comprised $49.5 \%$ of all sampled species.

In this study, we report 19 new occurrences phanerogamic species for Maranhão State. Among those, five species (Duguetia surinamensis, Dioclea reflexa, Utricularia fimbriata, Duroia macrophylla e Manilkara bidentata) have restricted distribution to the Amazon ecosystem, and three (Paepalanthus caldensis, Guilandina bonduc, Ouratea fieldingiana) to the Atlantic forest.

In terms of life forms, we identified 41 nanophanerophytes, 34 terophytes, 15 camephytes, ten microphanerophytes, six hemicryptophytes, five lianas, two vines, two hemiparasites, and one parasite (Figure 1).

Regarding the physiognomic classification of Sítio Aguahy, three physiognomies can be noticed, flooded fields, nonflooded shrubby and non-flooded forest.

Flooded fields comprise a predominant vegetation of herbs, such as Utricularia fimbriata, Utricularia nigrescens,

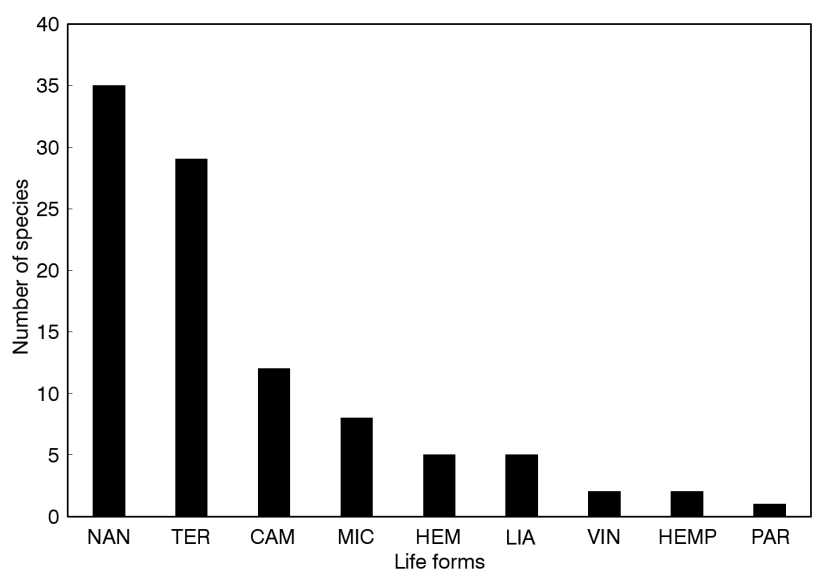

Figure 1. Number of species by life forms recorded in the Restinga of Sítio Aguahy, municipality of São José de Ribamar, Maranhão State, Brazil. Abbreviations: NAN - nanophanerophytes, TER - terophytes, CAM - camephytes, MIC - microphanerophytes, HEM - hemicryptophytes, LIA lianas, VIN - vines, HEMP - hemiparasites, and PAR - parasite. 
Paepalanthus bifidus, Paepalanthus lamarckii, Paepalanthus sublitis and Xyris jupicai, especially during the flood season. Non-flooded shrubby physiognomy counts on shrub species, e.g. Chrysobalanus icaco and Byrsonima crassifolia, which might present grouping formation like bushes or be associated to several individuals from the same species and other herbs, most likely with Cyperaceae and Poaceae representatives. Non-flooded forest physiognomy was observed in parallel to shrubby formation that occupies land until nearshore. In this system, $10 \mathrm{~m}$ height trees are common, such as Manilkara bidentata, Manilkara triflora and Tapirira obtusa. There are also few shrubs and small trees sparsely distributed closest to the shoreline with Guilandina bonduc and Chloroleucon acacioides representatives.

Similarity analysis identified three groups (Figure 2) with high levels of likeness (65\%). Cophenetic analysis demonstrated that the dendrogram adequately represented original data's matrix $(\mathrm{r}=0.97)$. One group was formed by this study (Aguahy site) and the restingas of Pará State (Algodoal 1 and Algodoal 2); a second group was formed by restinga areas on Ilha Grande, Parnaíba, and Luiz Correia (all in Piauí State); the Maiandeua site was the only one that displayed full dissimilarity from the others.

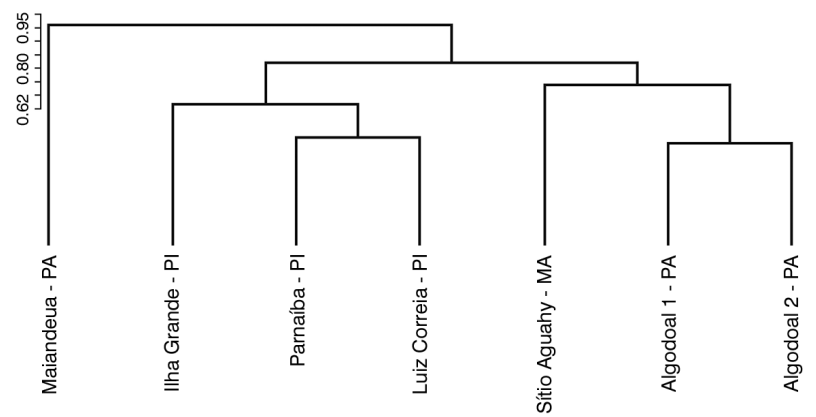

Figure 2. Dendrogram resulting from the floristic similarity analysis (Cluster) between floristic surveys of the Restinga of Sítio Aguahy, municipality of São José de Ribamar, Maranhão (this study), with other surveys in Restingas areas of Pará (PA) and Piauí (PI).

\section{DISCUSSION}

The families of greater richness in the restinga of this study were also commonly referenced in other studies of restingas in northeastern Brazil (Almeida Jr. et al. 2009; Cantarelli et al. 2012; Santos-Filho et al. 2013). In this context, special emphasis should be given to Fabaceae and Rubiaceae - that comprise a large number of species in lowland neotropical forests (Gentry 1988) - and Myrtaceae - in which the center for expansion is in humid forests of eastern Brazil, with representatives also later colonizing other habitats (Mori et al. 1983).
We hereby report our findings of 19 new botanical occurrences for Maranhão, including five species - Duguetia surinamensis, Dioclea reflexa, Utricularia fimbriata, Duroia macrophylla, and Manilkara bidentata - whose distribution was restricted to the Amazon forest biome, and two species associated with the Atlantic forest - Guilandina bonduc, Ouratea fieldingiana. Other species were previously known from other ecosystems, such as Caatinga and Cerrado (List of species of the Brazilian Flora 2014). This high number of new species occurrences exemplifies the scarcity of information available for restinga areas in Maranháo State, as it was also suggested by Zickel et al. (2004). This gap of floristic composition studies in restinga ecosystems directly affects development of detailed and deepened ecology assessment of these environments.

Life form data revealed that low-growing plants dominated vegetation in the study area, with predominant phanerophytes, but also with consistent presence of terophytes and camephytes. Similar life forms ratio were also observed in restinga areas in the states of Rio Grande do Norte (Almeida Jr. et al. 2006), Pernambuco (Almeida Jr. et al. 2007; Almeida Jr. et al. 2009) and Piauí (Santos-Filho et al. 2010; Santos-Filho et al. 2013). Almeida Jr. et al. (2009) and Santos-Filho et al. (2013) reported that soil type, soil nutrient levels, and depth of water table, directly influence the proportion of individuals of each life form.

Similarity between restinga flora of Sítio Aguahy and Algodoal 1 and Algodoal 2 in Pará State suggests the colonization of Maranhão coastal areas derived from the Amazon forest. However, in spite of this floristic likeness and the fact these areas are geographically close, these three floras show distinct species compositions that reflect unique abiotic and biotic interactions (Santos-Filho et al. 2013).

Amaral et al. (2008) noticed differences between restinga families' species richness throughout the Amazonian coast (Fabaceae, Cyperaceae, Poaceae, Rubiaceae, Myrtaceae and Asteraceae) and common families in the Atlantic Forest (families cited above) that were found in restinga sites along the coast of Rio de Janeiro State (Scarano 2002). A good example is Eriocaulaceae, among the ten families with greatest number of species along the coast of the Amazon region (Amaral et al. 2008 and present study), but was not listed even among the 20 most important families in a survey undertaken in Rio de Janeiro (Araujo 2000). Moreover, floristic surveys undertaken in northeastern Brazil often show the family Eriocaulaceae appearing among the 20 richest families (Sacramento et al. 2007; Almeida Jr. et al. 2009; Cantarelli et al. 2012), or completely absent in the list of species (Almeida Jr. et al. 2007; Santos-Filho et al. 2013). This scenario emphasizes the urgency for further studies in the area to ensure ultimate robust comparisons of coastal floras of Brazilian northeast region. 
Another interesting remark is that Manilkara bidentata and Manilkara cavalcantei Pires \& W. A. Rodrigues ex T. D. Penn. have been sampled along the coasts of Maranhão and Piauí respectively. Both taxa are preferentially Amazonian (Almeida Jr. et al. 2011) and their presence in restinga areas supports the hypothesis that coastal vegetation can provide ecological corridors in ecotone environments (Castro et al. 2012). This hypothesis can only be explored with intensive and long-term sampling effort along the coast of Maranháo, to elucidate how these species are distributed in the Brazilian coastal.

\section{CONCLUSIONS}

Although this study presents an initial survey of restinga vegetation in Maranhão State, the ecosystem showed high plant richness, and special attention should be given to the 19 new species occurrences presented here. The predominance of phanerophytes, therophytes, and camephytes show a distribution of the spectrum of species, similar to other restingas in northeastern Brazil. In addition, the greater floristic similarity amongst areas in the state of Maranhão and Pará indicate a possible influence of neighboring vegetation in the process of colonization of species in areas of restinga.

\section{ACKNOWLEDGEMENTS}

Our special thank you, to CNPq for the fellowship granted to the first author in Universidade Federal do Maranhão (UFMA); to Companhia Farmacêutica Quercegen Agronegócios I Ltda for access authorization to the area where the study was performed; David B. Muniz for assembling the map, Mariana Bonfim for collaboration in reviewing the text, and FAPEMA for financial support (Process 2 887/12).

\section{REFERENCES}

Almeida Jr., E.B.; Zickel, C.S.; Pimentel, R.M.M. 2006. Caracterização e espectro biológico da vegetação do litoral arenoso do Rio Grande do Norte. Revista de Geografia, 23: 45-58.

Almeida Jr., E.B.; Pimentel, R.M.M.; Zickel, C.S. 2007. Flora e formas de vida em uma área de restinga no litoral norte de Pernambuco, Brasil. Revista de Geografia, 24: 19-34.

Almeida Jr., E.B; Olivo, M.A; Araújo, E.L.; Zickel, C.S. 2009. Caracterização da vegetação de restinga da RPPN de Maracaípe, $\mathrm{PE}$, Brasil, com base na fisionomia, flora, nutrientes do solo e lençol freático. Acta Botanica Brasilica, 23: 36-48.

Almeida, Jr., E.B.; Santos-Filho, F.S.; Zickel, C.S. 2011. Magnoliophyta, Ericales, Sapotaceae, Manilkara cavalcantei Pires and Rodrigues ex T.D. Penn: First occurrence for northeastern Brazil. Checklist, 7: 53-54.

Amaral, D.D.; Prost, M.T.; Bastos, M.N.C.; Costa-Neto, S.V.; Santos, J.U.M. 2008. Restingas do litoral amazônico, estados do Pará e Amapá, Brasil. Boletim do Museu Paraense Emílio Goeldi Ciências Naturais, 3: 35-67.
APG III. 2009. An update of the Angiosperm Phylogeny Group classification for the orders and families of flowering plants: APG III. Botanical Journal of the Linnean Society, 161: 105-121.

Araujo, D.S.D. 2000. Análise floristica e fitogeográfica das restingas do Estado do Rio de Janeiro. Tese de Doutorado. Universidade Federal do Rio de Janeiro, Rio de Janeiro.

Araujo, D.S.D.; Henriques, R.P.B. 1984. Análise florística das restingas do estado do Rio de Janeiro. In: Lacerda, L.D.; Araujo, D.S.D.; Cerqueira, R.; Turcq, B. (Ed.). Restingas: origem, estrutura e processos. Niterói: CEUFF, p.47-60.

Araujo, A.C.M.; Silva, A.N.F.; Almeida Jr., E.B. 2016. Caracterização estrutural e status de conservação do estrato herbáceo de dunas da Praia de São Marcos, Maranhão, Brasil. Acta Amazonica, 46:

Bastos, M.N.C. 1988. Levantamento florístico em restinga arenosa litorânea na Ilha de Maiandeua-Pará. Boletim do Museu Paraense Emílio Goeldi, Série Botânica, 4: 159-176.

Bastos, M. N.C.; Rosário, C.S.; Lobato, L.C.B. 1995. Caracterização fitofisionômica da restinga de Algodoal, Maracanã-PA, Brasil. Boletim Museu Paraense Emilio Goeldi, Série Botânica, 11: 173-197.

Borcard, D.; Gillet, F.; Legendre, P. 2011. Numerical Ecology with $R$. Springer, New York. 306.

Cabral-Freire, M.C.; Monteiro, R. 1993. Florística das praias da Ilha de São Luís, Estado do Maranhão (Brasil): Diversidade de espécies e suas ocorrências no litoral brasileiro. Acta Amazonica, 23: 125-140.

Cantarelli, J.R.R.; Almeida Jr., E.B.; Santos-Filho, F.S.; Zickel, C.S. 2012. Tipos fisionômicos e flora vascular da restinga da APA de Guadalupe, Pernambuco, Brasil. Insula, 41: 95-117.

Castro, A.S.F.; Moro, M.F.; Menezes, M.D. 2012. O complexo vegetacional da zona litorânea no Ceará: Pecém, São Gonçalo do Amarante. Acta Botanica Brasilica, 26: 108-124.

Chaves, A.D.C.G.; Santos, R.M.D.S.; Santos, J.O.D.; Fernandes, A.D.A.; Maracajá, P.B. 2013. A importância dos levantamentos florístico e fitossociológico para a conservação e preservação das florestas. Agropecuária Cientifica no Semiarido, 9: 43-48.

Dias, H.M.; Soares, M.L.G. 2008. As Fitofisionomias das Restingas do Município de Caravelas (Bahia- Brasil) e os bens e serviços associados. Boletim técnico-científico do CEPENE, 16: 59-74.

Ellenberg, H.; Mueller-Dombois, D. 1967. A key to Raunkiaer plant life forms with revised subdivisions. Berichte des Geobotanischen Institutes der Eidg. Techn. Hochschule, Stiftung Rübel, 37: 56-73.

El-Robrini, M.; Marques, J.V.; Silva, M.M.A.; El-Robrini, M.H.S.; Feitosa, A.C.; Tarouco, J.E.F.; Santos, J.H.S.; Viana, J.S. 2006. Maranhão. In: D. Muehe (Ed.). Erosão e progradação do litoral brasileiro: Maranhão. Brasília, MMA. 2006. Access 14/10/2014.

Gentry, A.H. 1988. Changes in plant community diversity and floristic composition on environmental and geographical gradients. Annals of the Missouri Botanical Garden, 1-34.

IBGE - Instituto Brasileiro de Geografia e Estatística. 2012. Manual técnico da vegetação brasileira. Fundação do Instituto Brasileiro de Geografia e Estatística. Rio de Janeiro. 275p.

INMET - Instituto de Meteorologia. 2015. Climatologia (http:// www.inmet.gov.br/ portal/index.php?r=clima/mês Tempo). Acesso em: 29/12/2015. 
Köppen, W. 1948. Climatologia: con un estudio de los climas de la terra. México. Fondo de Cultura Económica, México. 479p.

List of species of the Brazilian Flora. 2014. Rio de Janeiro Botanical Garden. Available in http://floradobrasil.jbrj.gov.br. Access on 14 Fev 2015.

Mori, S.A.; Boom, B.M.; Carvalino, A.M.; Santos, T.S. 1983. Ecological importance of Myrtaceae in an eastern Brazilian wet forest. Biotropica, 15: 68-70.

Mori, L.A.; Silva, L.A.M.; Lisboa, G.; Coradin, L. 1989. Manual de manejo do herbário fanerogâmico. Ilhéus, Centro de Pesquisa do Cacau. 97p.

Oksanen, J.; Blanchet, F.G.; Kindt, R.; Legendre, P.; Minchin, P.R.; O’Hara, R.B.; Simpson, G.L.; Solymos, P.; Stevens, M.H.H.; Wagner, H. 2013. Package 'vegan': Community Ecology Package. $\mathrm{R}$ package version 2.0.10.

Raunkiaer, C. 1934. The life forms of plants and statistical plant geography. Oxford, Clarendon. 632p.

R Development Core Team. 2013. R: A Language and Environment for Statistical Computing version 3.1.1. Foundation for Statistical Computing. Viena.

Sacramento, A.C.S.; Zickel, C.S.; Almeida Jr., E.B. 2007. Aspectos florísticos da vegetação de restinga no litoral de Pernambuco. Revista Árvore, 31: 1121-1130.

Santos-Filho, F.S.; Almeida Jr., E.B.; Soares, C.J.R.S. ; Zickel, C.S. 2010. Fisionomias das restingas do Delta do Parnaíba, Nordeste, Brasil. Revista Brasileira de Geografia Física, 3: 218-227.
Santos-Filho, F.S.; Almeida Jr., E.B; Zickel, C.S. 2013. A flora das restingas de Parnaíba e Luiz Correia - litoral do Piauí, Brasil. In: Santos-Filho, F.S; Leite Soares, A.F.C; Almeida Jr., E.B. (Ed.). Biodiversidade do Piaui: pesquisas \& perspectivas vol. 2. Curitiba: CRV. p. 36-59.

Santos-Filho, F.S.; Almeida Jr., E.B.; Soares, C.J.R.S.; Zickel, C.S. 2015. Flora and woody vegetation structure in an Insular area of restinga in Brazil. International Journal of Ecology and Environmental Sciences, 41(3-4): 147-160.

Santos, J.U.M.; Rosário, C.S. 1988. Levantamento da vegetação fixadora de dunas de Algodoal-PA. Belém. Boletim do Museu Paraense Emílio Goeldi, Série Botânica, 4: 133-151.

Scarano, F.R. 2002. Structure, function and floristic relationships of plant communities in stressful habitats marginal to the Brazilian Atlantic rainforest. Annals of Botany, 90: 517-524.

Silva, S.M.; Britez, R.M. 2005. A vegetação da planície costeira. In: Marques, M.C.M.; Britez, R.M. (Orgs.). História Natural e Conservação da Ilha do Mel. Curitiba, Ed. da UFPR, Curitiba, Paraná, Pp. 49-84.

Souza, C.R.G.; Hiruma, S.T.; Sallun, A.E.M.; Ribeiro, R.R.; AzevedoSobrinho, J.M. 2008. "Restinga": Conceitos e Empregos do Termo no Brasil e Implicaçôes na Legislação Ambiental. Instituto Geológico - Secretaria de Meio Ambiente do Estado de São Paulo. 104p.

Zickel, C.S.; Vicente, A.; Almeida Jr., E.B.; Cantarelli, J.R.R.; Sacramento, A.C. 2004. Flora e vegetação das restingas no Nordeste Brasileiro. In: Eskinazi-Leça, E.; Neumann-Leitão, S.; Costa, M.F. (Ed.). Oceanografia: um cenário tropical. Recife: Bargaço. p. 689-701.

Recebido em 22/10/2015

Aceito em 02/03/2016 
